\title{
Calidad Acústica de Aulas Universitarias: Análisis y Evaluación de Parámetros Acústicos de Recintos
}

\section{Acoustic Quality of University Classrooms: Analysis and Evaluation of Acoustic Parameters of Venues}

\section{Sebastián P. Ferreyra}

Centro de Investigación y Transferencia en Acústica (CINTRA), UA CONICET Facultad Regional Córdoba, Universidad Tecnológica Nacional

sferreyra@frc.utn.edu.ar

\section{Gabriel A. Cravero}

Centro de Investigación y Transferencia en Acústica (CINTRA), UA CONICET Facultad Regional Córdoba, Universidad Tecnológica Nacional gacravero@gmail.com

\section{Hugo C. Longoni}

Centro de Investigación y Transferencia en Acústica (CINTRA), UA CONICET Facultad Regional Córdoba, Universidad Tecnológica Nacional hlongoni@frc.utn.edu.ar

\section{Juan F. López}

Centro de Investigación y Transferencia en Acústica (CINTRA), UA CONICET Facultad Regional Córdoba, Universidad Tecnológica Nacional facundolo01@gmail.com

\section{Manuel F. Parada}

Centro de Investigación y Transferencia en Acústica (CINTRA), UA CONICET Facultad Regional Córdoba, Universidad Tecnológica Nacional manuelfparada@gmail.com

\section{Marcos S. Díaz}

Centro de Investigación y Transferencia en Acústica (CINTRA), UA CONICET Facultad Regional Córdoba, Universidad Tecnológica Nacional

marcossantiagodiaz@hotmail.com 


\title{
Resumen
}

En nuestro país, por lo general, las aulas han sido construidas sin considerar criterios acústicos, generando ambientes inadecuados para el proceso de enseñanza-aprendizaje. Esta situación, interfiere tanto en el desempeño académico de los alumnos, como en la salud de los docentes. En este trabajo se analizan los principales factores acústicos intervinientes en dicho proceso, tales como: geometría y materiales del recinto, ruido de fondo, tiempos de reverberación y relación señal/ruido. Los resultados evidencian que una cantidad significativa de aulas utilizadas en el dictado de carreras de grado no presentan condiciones adecuadas para el desarrollo del proceso de enseñanza-aprendizaje. Los resultados presentados se obtuvieron en el marco del PID UTN 1658: "Estudio de características principales del campo sonoro en aulas y auditorios.

Palabras Clave: Acústica de Aulas, Ruido de Fondo, Tiempo de Reverberación, Relación Señal/ Ruido.

\begin{abstract}
In our country, in general, classrooms have been built without considering acoustics criteria, resulting in inadequate environments for teaching and learning process. This situation interferes both in the academic performance of students and the health of teachers. In this paper the main acoustic factors involved in this process are analyzed, such as: geometry and material of the enclosure, background noise, reverberation time and signal to noise ratio. The results show that a significant number of classrooms used in the dictation of degree courses do not present adequate conditions for the development of the teaching-learning process. Submitted results were obtained under the UTN PID 1658: "Study of main characteristics of the sound field in classrooms and auditoriums".
\end{abstract}

Keywords: Classroom Acoustics, Background Noise, Reverberation Time, Signal/Noise Ratio.

\section{INTRODUCCIÓN}

Una serie de fenómenos acústicos se desencadenan cuando una onda sonora entra en contacto con superficies y objetos de un recinto. Estos fenómenos son la reflexión, refracción y difusión.

Las ondas sonoras emitidas por una fuente acústica se ven modificadas en su dirección, espectro y magnitud por el medio de propagación (aire principalmente), medios sólidos como paredes, techo y otros objetos. Asumiendo que existe un camino sin obstrucción entre la fuente y un oyente, éste escuchará primero el sonido directo, seguido de las reflexiones tempranas, especulares y difusas producidas por las superficies más cercanas y luego las 
reflexiones tardías o cola de reverberación. La respuesta impulsiva del recinto (RIR) o ecograma, caracteriza en el dominio del tiempo la transmisión de las ondas sonoras para una ubicación particular de fuente-receptor.

Un aspecto importante en el estudio de la audición espacial, es el efecto de las reflexiones sobre la habilidad para ubicar y discriminar una fuente sonora. El sistema auditivo se enfrenta con un conglomerado de información sonora y debe ser capaz de resolver la competencia perceptual que se produce entre la fuente sonora original y sus reflexiones. Fenómenos como el excesivo ruido ambiente y elevada reverberación, interfieren en la comunicación en recintos destinados al proceso de enseñanza-aprendizaje, provocando en sus actores desagrado, malestar, cansancio, falta de concentración y frecuentemente, enfermedades fonoaudiológicas (WHO, 2001).

En nuestro país, por lo general, las aulas han sido construidas sin considerar criterios acústicos, generando ambientes inadecuados para el proceso de enseñanza-aprendizaje (Ercoli et al, 2001). Los resultados presentados en este artículo se obtienen en el marco del PID UTN N ${ }^{\circ}$ 1658: "Estudio de características principales del campo sonoro en aulas y auditorios”. El propósito de dicho proyecto es analizar las características acústicas de recintos destinados a proceso de enseñanza-aprendizaje, abarcando aulas y pequeños auditorios utilizados en el nivel universitario, mediante métodos objetivos (mediciones físicas) y subjetivos (pruebas perceptuales con participación de estudiantes, docentes y no docentes), con la finalidad de formular propuestas destinadas a establecer criterios de diseño y calidad acústica de recintos. Para alcanzar los objetivos, se planteó realizar una serie de ensayos objetivos basados en el análisis de parámetros e indicadores recomendados en la norma ISO 3382, derivados de la RIR. Como también, pruebas perceptuales que consisten en la determinación de la inteligibilidad del habla, utilizando principios de realidad acústica virtual que facilitarán tanto el estudio auditivo como el acústico físico de los recintos.

\begin{tabular}{|l|l|l|l|l|l|}
\hline Recinto & $\begin{array}{l}\text { Vol. } \\
{\left[\mathrm{m}^{3}\right]}\end{array}$ & $\begin{array}{l}\text { Largo } \\
{[\mathrm{m}]}\end{array}$ & $\begin{array}{l}\text { Ancho } \\
{[\mathrm{m}]}\end{array}$ & $\begin{array}{l}\text { Alto } \\
{[\mathrm{m}]}\end{array}$ & Capacidad \\
\hline AU011 & 93,45 & 6,94 & 4,44 & 3,02 & $20^{\text {bancos }}$ \\
\hline AU154 & 143,32 & 7,05 & 6,98 & 2,90 & $51^{\text {bancos }}$ \\
\hline AU608 & 179,46 & 8,30 & 5,94 & 3,63 & $35^{\text {bancos }}$ \\
\hline AU015 & 194,25 & 10,00 & 7,00 & 2,90 & $54^{\text {bancos }}$ \\
\hline AUD2 & 215,60 & 10,63 & 6,97 & 2,91 & $60^{\text {butacas }}$ \\
\hline AU225 & 240,45 & 11,92 & 6,72 & 2,98 & $98^{\text {bancos }}$ \\
\hline AU708 & 318,82 & 12,17 & 7,66 & 3,42 & $117^{\text {bancos }}$ \\
\hline AU405 & 561,02 & 16,06 & 11,35 & 3,11 & $216^{\text {bancos }}$ \\
\hline AUD1 & 712,70 & 17,44 & 10,7 & 3,83 & $168^{\text {butacas }}$ \\
\hline
\end{tabular}

Tabla 1: Características de las aulas y auditorios.

En este proyecto de carácter experimental se pudo demostrar a través de la medición de parámetros objetivos (derivados de la RIR y el ruido de fondo) que las características del campo sonoro en la población de aulas estudiadas en general no son adecuadas para el 
desarrollo del proceso enseñanza-aprendizaje.

El trabajo está organizado de la siguiente manera: en primer lugar, se sintetiza el resultado del relevamiento acústico arquitectónico, se presenta la metodología y resultados de la medición de parámetros acústicos derivados de la RIR y ruido de fondo. En segundo lugar, se realiza el análisis y discusión de los resultados obtenidos. Finalmente, se presentan las conclusiones y trabajos a futuro.

\section{TRABAJO EXPRIMENTAL}

\section{Relevamiento acústico arquitectónico}

El relevamiento de características acústicas arquitectónicas se realizó para una muestra de 9 recintos (7 aulas y 2 auditorios) de la Facultad Regional Córdoba, Universidad Tecnológica Nacional (FRC, UTN) (Cravero et al 2013, Cravero et al 2014, Longoni et al 2014). El criterio de selección consideró características constructivas, geometría, materiales y entorno próximo de cada aula, resultado representativa para una población de 87 aulas y 2 auditorios. En la Tabla 1 se presentan las dimensiones y la capacidad de cada Aula (AU) y Auditorio (AUD) de la muestra. El relevamiento realizado, evidencia que el 80,9 \% de los recintos presentan materiales con bajos coeficientes de absorción sonora ( $(\alpha)$, tales como hormigón, baldosa de granito, vidrio, ladrillo revocado y pintado, placas de roca yeso, entre otros. Mientras que el 19,1 \% restante verificó la existencia de materiales con valores medios y altos de a, utilizados principalmente en cielorraso, paredes y piso.

Se destaca que, el AUD2 fue diseñado y construido considerando criterios acústicos, presentando cielorraso de placas de roca-yeso de $12,5 \mathrm{~mm}$ de espesor con perforaciones cuadradas y fieltro de lana de vidrio; paredes revestidas con placas de madera enchapada de 19 mm y piso de madera recubierto con alfombra tipo buclé. El 80,9 \% de las aulas seleccionadas son utilizadas para el dictado de clases en carreras de grado, mientras que el 16,6 \%, para posgrado y extensión universitaria. La representatividad de la muestra respecto a la población se ilustra en Fig. 1.

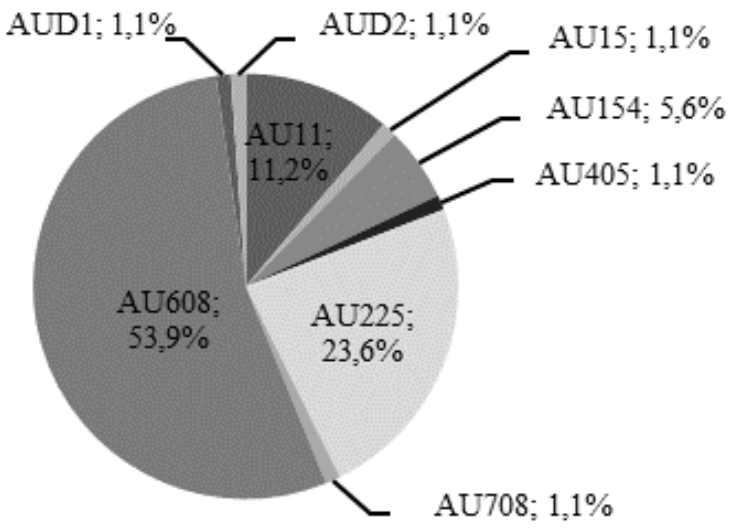

Fig.1 Representatividad de la muestra. 


\section{Metodología de medición de RIR}

La RIR caracteriza completamente la propagación de las ondas sonoras para cada posición de la fuente y el receptor. La medición de la RIR se realizó siguiendo las directrices de la norma IRAM 4109-2 (equivalente a ISO 3382-2), utilizando un sistema electroacústico optimizado para tal fin (Ferreyra et al, 2009). Dicho sistema está compuesto por un micrófono electroestático de medición $B \& K$ 4133, un preamplificador para micrófono de medición B\&K 2804, un simulador de cabeza y torso artificial B\&K 4128, un amplificador de audio de potencia Samsom Servo 300, un sistema de gestión de altavoces dBx Driverack PA+, una fuente sonora omnidireccional tipo dodecaedro, un sistema de adquisición de sonido portable Presonus Audio Vox 44VSL, programas de acústica de salas B\&K 7841 y SAMSoft, auriculares Sennheiser HD280pro, distanciómetro laser Leica D5 y un computador personal portable equipado con microprocesador Intel i3 2300, RAM 4GB, HD 500 GB. Además, las mediciones de niveles sonoros se realizaron con medidores de nivel de presión sonora integradores B\&K 2250 y RION NL-31 equipados con filtros digitales normalizados para el análisis de frecuencia y ambos calibrados al inicio y fin de las mediciones. Estás últimas se efectuaron según procedimientos de medición ad hoc del CINTRA desarrollados por el equipo de investigación.

La RIR fue obtenida aplicando la técnica de deconvolución de señales determinísticas, utilizando como excitación un barrido sinusoidal de variación exponencial de $21 \mathrm{~s}$ de duración. Se registraron un total de 12 RIR correspondientes a 6 posiciones de micrófono y 2 posiciones de fuente en cada recinto.

\section{Parámetros derivados de la RIR}

a) Tiempo de reverberación

El tiempo de reverberación, $\mathrm{T}$ se define como el tiempo transcurrido desde que la fuente sonora cesa su emisión hasta el instante en que el nivel de presión sonora decae $60 \mathrm{~dB}$ respecto de su valor inicial. Su medición de forma directa, presenta una gran dificultad, debido al elevado rango dinámico que debe tener el sistema de medición (superior a $60 \mathrm{~dB}$ ). Por esto último, se definieron diferentes parámetros (T20, T30) que permiten su cálculo para el caso de menor rango dinámico mediante extrapolación de los datos. El parámetro seleccionado para la determinación del tiempo de reverberación fue T30 propuesto por M. Schroeder (1965), calculado entre -5 y -35 dB de la curva de decrecimiento energético, la cual se obtiene a partir de la RIR. En las Fig. 2 y 3 se presentan los resultados del parámetro T30 con una resolución de octavas, en un ancho de banda en baja frecuencia (BF) de $31,5 \mathrm{~Hz}$ a $500 \mathrm{~Hz}$ y en alta frecuencia (AF) de $1 \mathrm{kHz}$ a $16 \mathrm{kHz}$. En la muestra AUD2 el T30 toma valores cercanos a $0,5 \mathrm{~s}$ en BF (Fig. 2) manteniendo este valor sin desvíos significativos en todo el ancho de banda (Fig. 3), lo cual evidencia el resultado óptimo de los criterios de diseño acústico aplicados en su construcción. Por otra parte, para el caso del AU15 los valores se mantienen en el orden de $2 \mathrm{~s}$ presentando baja dispersión en el espectro de BF, sin embargo el resto de los recintos evidencia desvíos significativos para el espectro de BF.

Cabe mencionar que el tiempo de reverberación es el único de los parámetros derivados de la RIR que actualmente posee valores recomendados para el diseño acústico de aulas. 


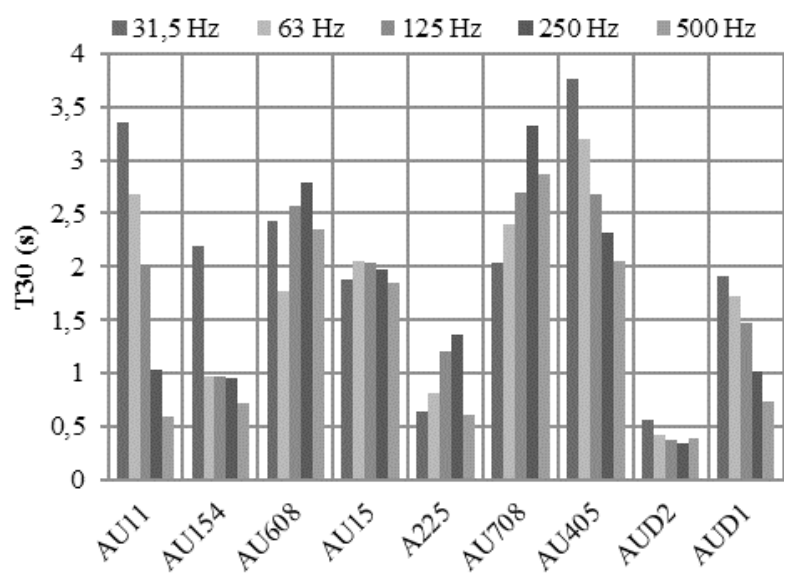

Fig. 2 - Tiempo de reverberación - T30 BF.

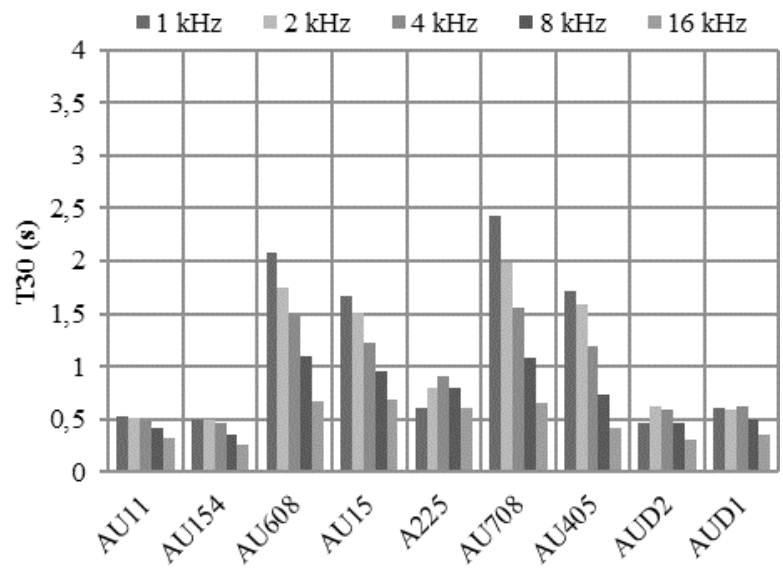

Fig. 3 - Tiempo de reverberación - T30 AF.

A nivel internacional existen normas que recomiendan un rango de valores de tiempo de reverberación adecuados para espacios destinados al proceso de enseñanza-aprendizaje. La Norma ANSI S12.60:2002, destinada para el nivel educativo primario, recomienda un valor T de $0,6 \mathrm{~s}$ para aulas para volúmenes menores a $283 \mathrm{~m} 3$ y de $0,7 \mathrm{~s}$ en volúmenes entre $283 \mathrm{~m} 3$ y $566 \mathrm{~m} 3$. Estos valores están definidos únicamente para las frecuencias de banda de octava de 500, 1000 y $2000 \mathrm{~Hz}$. 


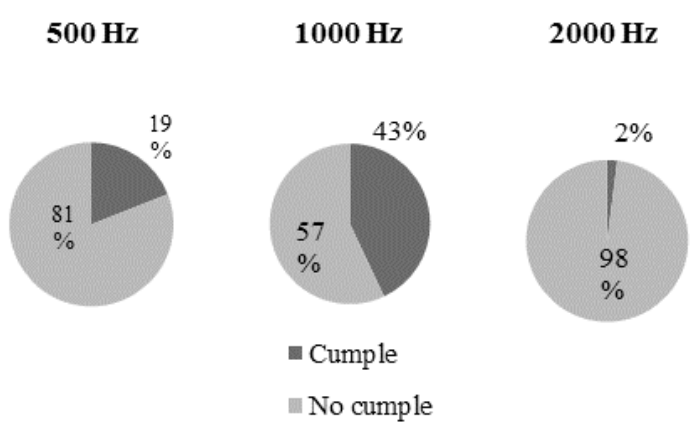

Fig. 4 - Evaluación T según norma ANSI S12.60:2002.

Los resultados de la evaluación según las directrices y recomendaciones de éste estándar se presentan en la Fig. 4. Se observa que para la frecuencia de $2 \mathrm{kHz}$, solamente el $2 \%$ presenta valores de T inferiores a los recomendados por ANSI S12.60.

\section{b) Tiempo de Decrecimiento Temprano}

El tiempo de decrecimiento temprano (EDT, por sus siglas en inglés) es el parámetro objetivo que mejor se correlaciona con la percepción del tiempo de reverberación. Jordan V. L. (1981) demuestra que la percepción de la reverberación se correlaciona mejor con la porción inicial de la curva de decrecimiento, determinando el EDT con la pendiente definida entre $0 \mathrm{~dB}$ y $-10 \mathrm{~dB}$ de la curva integrada de Schroeder.

En las Fig. 5 y 6 se presentan los resultados del EDT para BF y AF. Se observa que el comportamiento del EDT es comparable con T30, sin embargo es importante destacar que en algunos casos como el AU225 en la banda de frecuencia de $1 \mathrm{kHz}$ el EDT es un $20 \%$ menor que el T30.

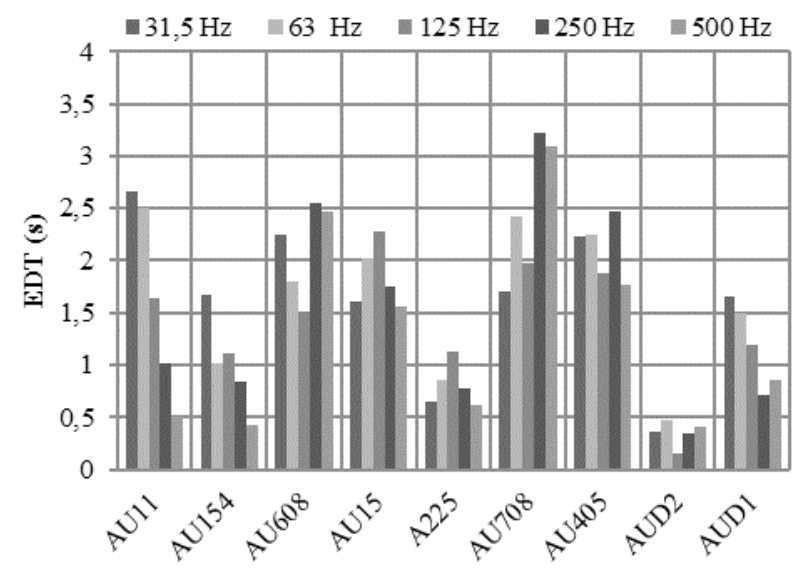

Fig. 5 - Tiempo de decrecimiento temprano - EDT BF 


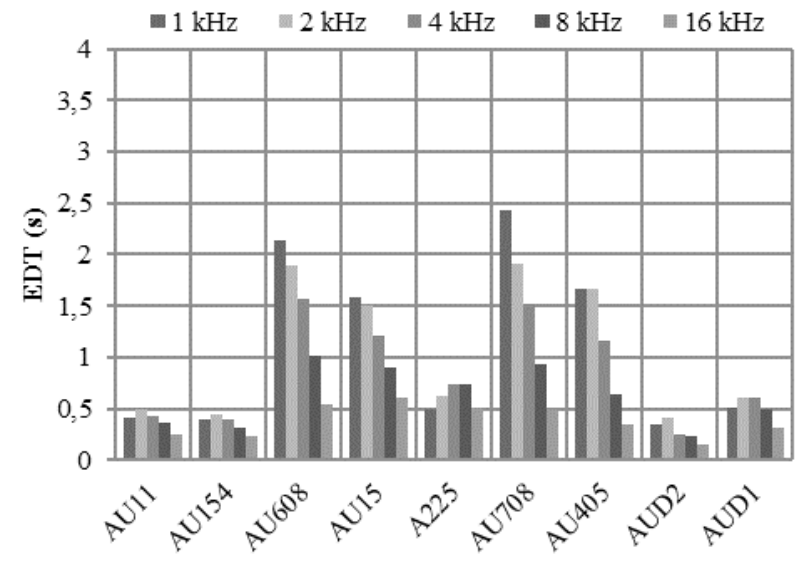

Fig. 6 - Tiempo de decrecimiento temprano - EDT AF.

c) Índice Energético Temprano Tardío (Claridad).

El índice energético temprano tardío, también llamado Claridad (Ct), propuesto por Richardt et al (1975), es un indicador de la relación entre la energía temprana y la tardía de la RIR. Se define como 10 veces el logaritmo de la razón entre la energía que arriba a un oyente dentro de los primeros "t" mili segundos desde la llegada del sonido directo (incluyéndolo) y la energía que lo alcanza con posterioridad.

En las Fig. 7 y 8 se presentan los resultados de C50 para el conjunto de mediciones realizadas. Donde 50 representa el valor de tiempo transición temprano-tardío expresado en ms. En la Fig. 7 se observa como este parámetro toma valores por debajo de $0 \mathrm{~dB}$ en todo el espectro de BF en el AU708 manteniendo valores cercanos a $0 \mathrm{~dB}$, en $\mathrm{AF}$, siendo esta última el aula con mayores valores de tiempo de reverberación. En el caso de AUD2 toma valores entre 8 y $18 \mathrm{~dB}$ en $\mathrm{AF}$ y entre 4 y $10 \mathrm{~dB}$ en $\mathrm{BF}$.

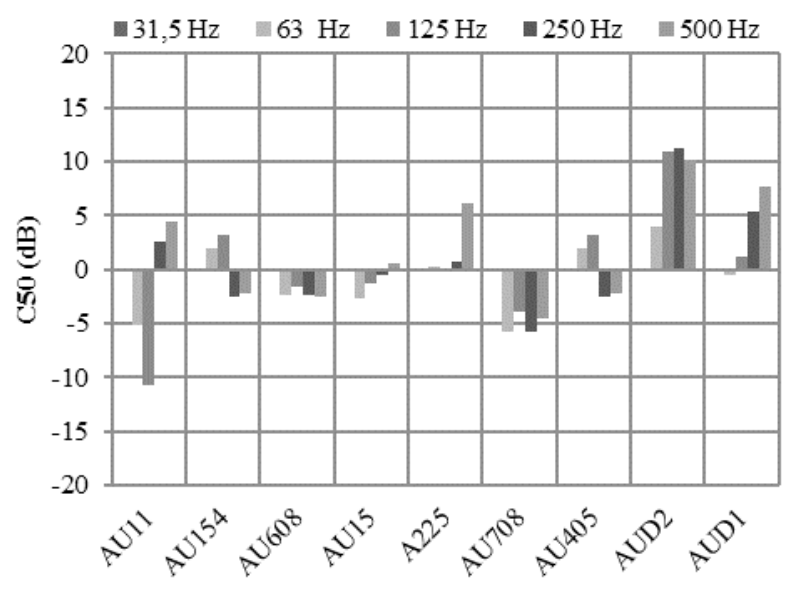

Fig. 7 - Índice Energético Temprano Tardío - C50 BF 


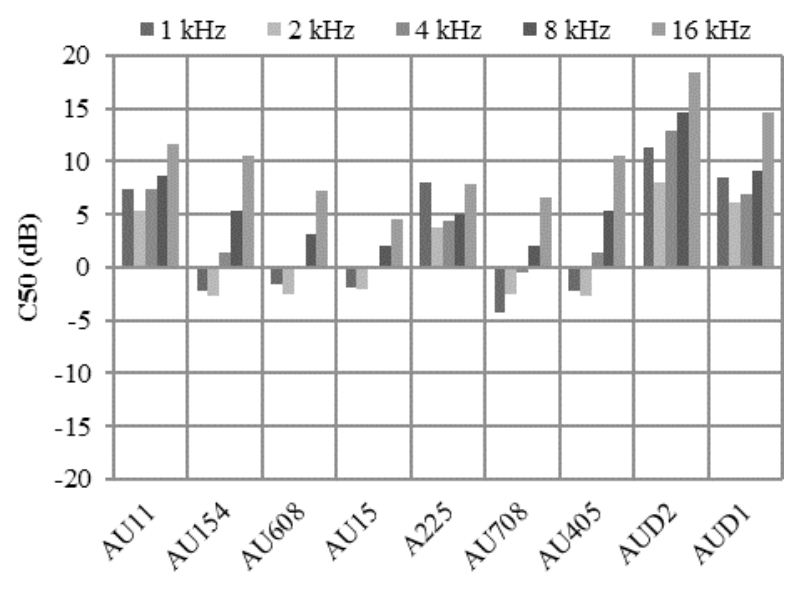

Fig. 8 - Índice Energético Temprano Tardío - C50 AF

\section{d) Definición}

Thiele (1953) propone un descriptor denominado D (del alemán Deulichkeit, que significa "claridad", "transparencia”). Éste se define como la relación entre la energía que llega al oyente dentro de los primeros $50 \mathrm{~ms}$, desde la llegada del sonido directo (incluyendo el sonido directo y las primeras reflexiones), y la energía total recibida.

En las Fig. 9 y 10 se presentan los resultados del parámetro D. En la Fig. 9 se observa una gran dispersión del parámetro D50 para BF en el AU11, con valores entre $10 \%$ y $80 \%$, mientras que en AF (Fig. 10) se mantiene entre $70 \%$ y $90 \%$. En el caso del AUD2, este parámetro se mantiene entre $75 \%$ y $100 \%$ en todo el espectro de frecuencias.

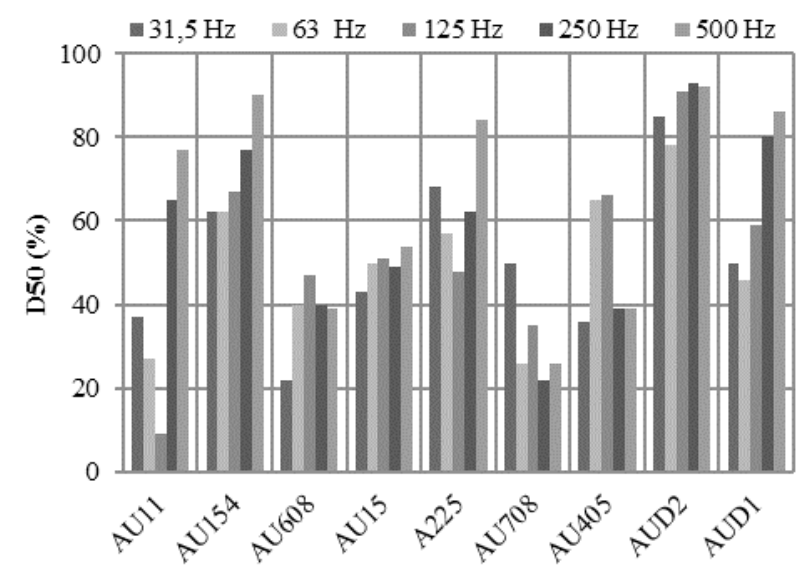

Fig. 9 - Definición - D50 BF 


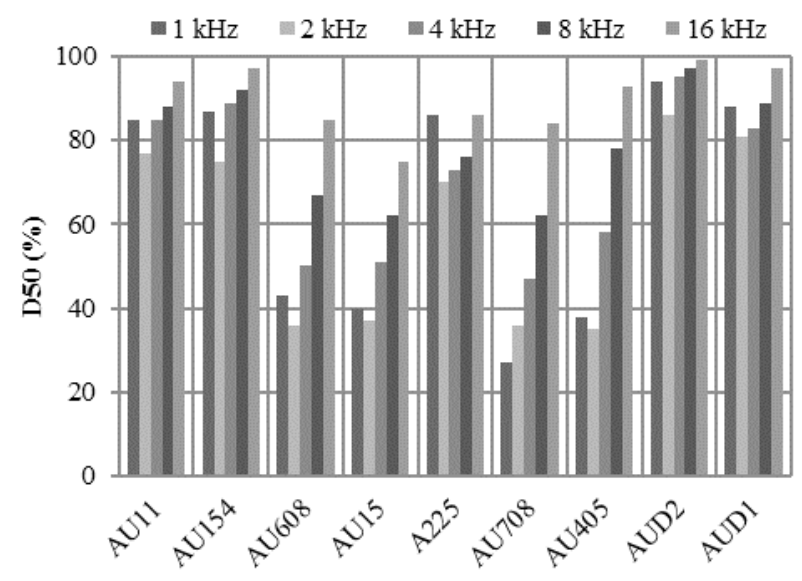

Fig. 10 - Definición - D50 AF.

\section{e) Centro de Tiempo}

Kürer, R., (1969) define al Centro de tiempo, ts como el momento de primer orden del área situada por debajo de la curva de decrecimiento energético. Este parámetro es indicativo del tiempo transcurrido desde el origen temporal $(t=0)$ hasta el "centro de gravedad" del área de la curva de decrecimiento energético. Por lo cual, la energía sonora medida desde el inicio hasta el valor de ts, es equivalente a la energía sonora medida desde el valor de ts hasta el fin de la curva de decrecimiento.

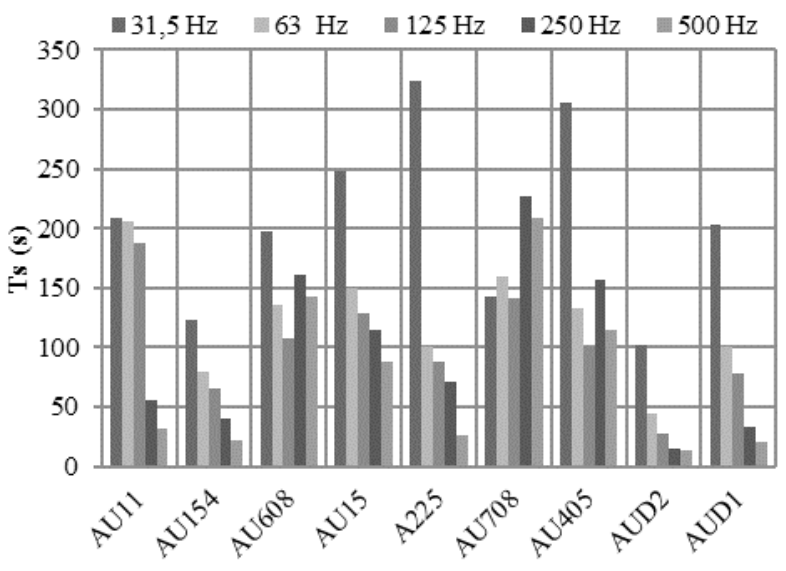

Fig. 11 - Centro de tiempo - Ts BF

En las Fig. 11 y 12 se presentan los resultados de este parámetro. Se observa que, en general, el ts presenta una mayor dispersión de valores en BF que en AF. Además, los valores en $\mathrm{BF}$ tienden a disminuir a medida que aumenta la frecuencia, mostrándose como una 
característica común a todos los recintos.

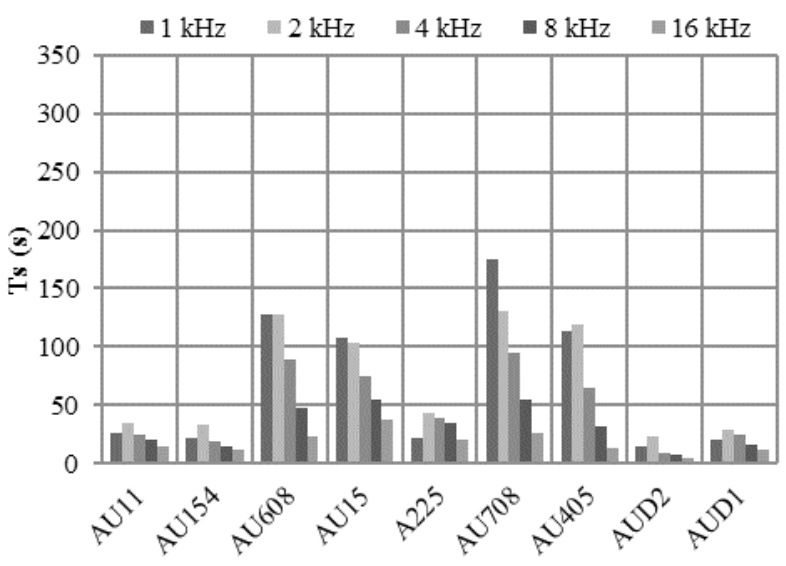

Fig. 12 - Centro de tiempo - Ts AF.

\section{f) Coeficiente Interaural de Correlación Cruzada}

El Coeficiente Interaural de Correlación Cruzada (IACC) fue propuesto por Yoichi Ando (1985), éste se define como la correlación cruzada entre las respuestas impulsivas de presión medidas en ambos oídos. El mismo es indicativo del grado de similitud existente entre ambas señales. Por lo cual el IACC asume valores entre 0 (correlación nula) y 1 (correlación total).

En las Fig. 13 y 14 se presentan los resultados del IACC medidos utilizado un simulador de cabeza y torso B\&K 4128. Se observa que este parámetro tiene valores cercanos a la unidad en $\mathrm{BF}$ y va decreciendo a medida que la frecuencia aumenta.

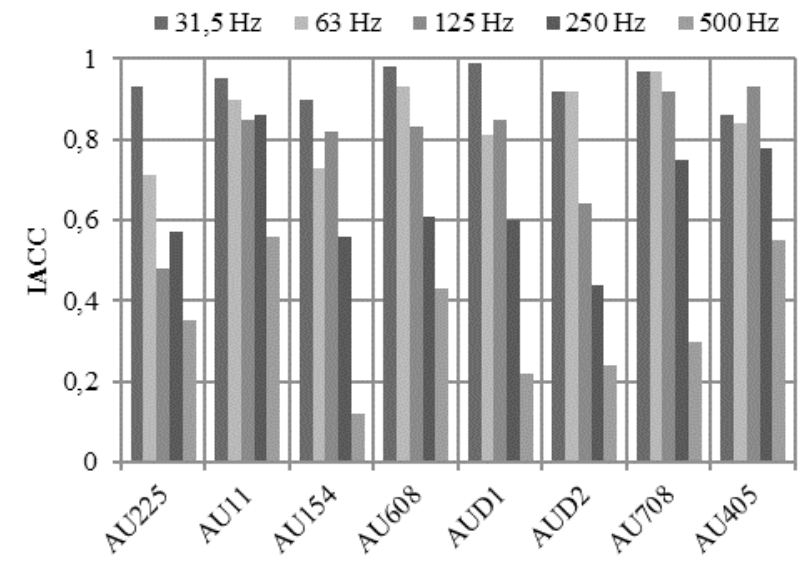

Fig. 13 - Coeficiente Interaural de Correlación Cruzada - IACC BF. 


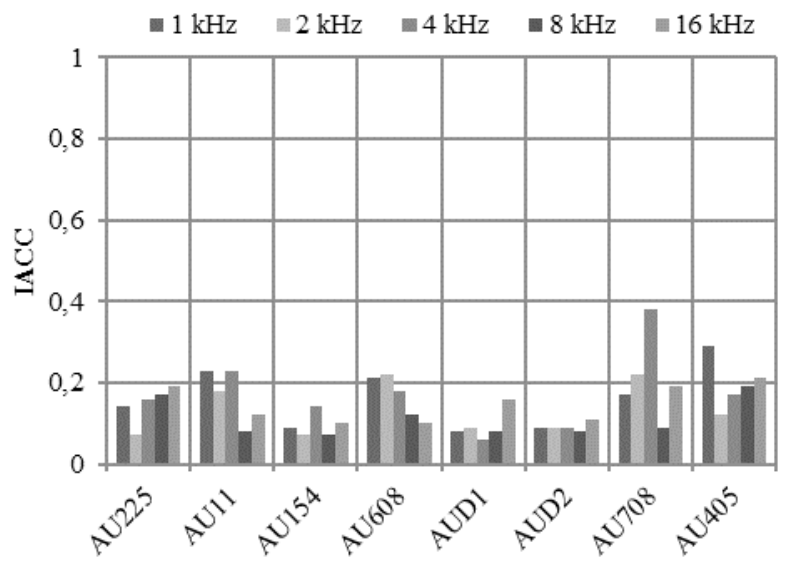

Fig. 14 - Coeficiente Interaural de Correlación Cruzada, IACC AF.

\section{Ruido de Fondo}

\section{a) Niveles globales de ruido de fondo}

En nuestro país actualmente no existe norma específica que recomiende valores globales máximos de ruido de fondo espacios donde se desarrolle el proceso de enseñanza-aprendizaje en función del nivel educativo y volúmenes de las aulas. Por tal motivo, para la evaluación de los valores globales de ruido de fondo de la muestra, se aplicaron las recomendaciones dadas por Building Bulletin 93 (Reino Unido), ANSI S12.60 (Estados Unidos) y NBR 10152 (Brasil).

La norma Building Bulletin 93:2003 establece un único valor global de ruido de fondo continuo equivalente (Leq) de $35 \mathrm{dBA}$, sin especificaciones del volumen de los recintos, aplicable en aulas de nivel primario y secundario. Por otra parte, la norma ANSI S12.60 recomienda para aulas de nivel primario valores de $35 \mathrm{dBA}$, en recintos de hasta $566 \mathrm{~m} 3$ y $40 \mathrm{dBA}$ para volúmenes mayores. Finalmente, la norma NBR 10152:1987 establece valores compatibles con el confort acústico en diversos ambientes. Para el caso de aulas y laboratorios en ambientes escolares, califica como confortables niveles de ruido de fondo menores a $40 \mathrm{dBA}$ y define un rango entre $40 \mathrm{dBA}$ y $50 \mathrm{dBA}$ como condición aceptable; midiéndose para todos los casos el Leq. En la Tabla 2 se presentan los resultados de las mediciones del nivel global de ruido de fondo ponderado A para la muestra (Longoni et al, 2014). La condición de los recintos fue: sala desocupada, puertas y ventanas cerradas, sistemas de ventilación y aire acondicionado (SVyAA) apagados, para un período de integración de $300 \mathrm{~s}$. En la Fig. $15 \mathrm{se}$ ilustra la representatividad de cada muestra (7) sobre la población total (89) para el estudio de ruido de fondo.

Al comparar los valores de la Tabla 2 para las condiciones SVyAA encendido y apagado se obtuvieron las siguientes diferencias: el 27,0 \% de la población mostró una diferencia entre 0 y $5 \mathrm{dBA}$, el 6,7 \% entre 5 y $10 \mathrm{dBA}$, el 1,2 \% entre 10 y $15 \mathrm{dBA}$ y el 55,1 \% entre 15 y $20 \mathrm{dBA}$. Se observa que el 63,3\% evidencia una diferencia de nivel de presión sonora superior a 10 dBA la cual perceptualmente resulta significativa.

La Fig. 16-a ilustra la distribución de los niveles de ruido de fondo medidos. De la evaluación de estos niveles resultó que ninguno de los recintos cumple con los criterios de 
Building Bulletin 93; solamente el AUD1 cumple con los criterios de la norma ANSI S12.60; el 12,4 \% cumple con los criterios de la norma NBR 10152, mientras que el resto $(87,6 \%)$ presenta niveles de ruido de fondo aceptables según lo establecido en esta última.

En la Fig. 16-b se presenta la distribución de los niveles globales de ruido de fondo para la condición de SVyAA encendidos. Se destaca que el AUD2 presentó 41,0 dBA, el nivel más bajo de la población.

\begin{tabular}{|c|c|c|c|}
\hline \multirow{2}{*}{ Recinto } & SVyAA & SVyAA & Diferencia de nivel \\
& Apagado & Encendido & [dBA] \\
\hline AUD1 & $\mathrm{L}_{\mathrm{eq}}[\mathrm{dBA}]$ & $\mathrm{L}_{\mathrm{eq}}[\mathrm{dBA}]$ & 15,5 \\
\hline AU11 & 36,9 & 52,4 & 10,3 \\
\hline AU608 & 38,8 & 49,1 & 18,4 \\
\hline AUD2 & 40,6 & 59,0 & 0,2 \\
\hline AU154 & 40,8 & 41,0 & 7,9 \\
\hline AU405 & 42,4 & 50,3 & 8,4 \\
\hline AU225 & 47,7 & 56,2 & 4,6 \\
\hline
\end{tabular}

Tabla 2 - Niveles globales de ruido de fondo ponderado A.
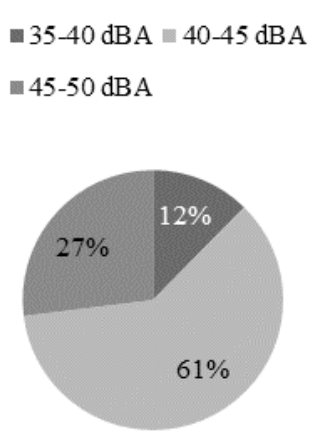

(a)
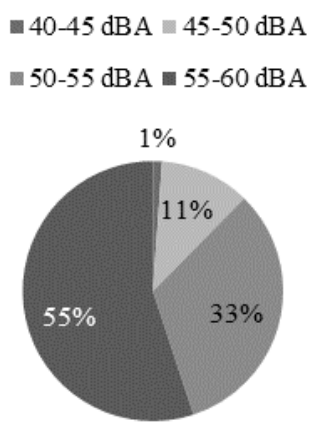

(b)

Fig. 16 - Distribución de niveles globales de ruido de fondo continúo equivalente para condiciones de SVyAA apagado (a) y encendido (b).

b) Perfiles de ruido de fondo 
En nuestro país, la Norma IRAM 4070 establece métodos de evaluación del espectro de ruido mediante un único valor numérico, por su contraste con una de las familias de curvas denominadas perfiles NC (noise criteria) y RC (room noise criterion), permitiendo calificar al ruido de fondo de un recinto como aceptable. La norma recomienda valores de referencia del espectro del ruido de fondo para un recinto según la actividad que en él se desarrolle. El procedimiento de cálculo de perfiles $\mathrm{NC}$, considera que para un recinto existente se debe obtener por mediciones de nivel sonoro continuo equivalente lineal, el espectro de bandas normalizadas de octava para frecuencias centrales desde $63 \mathrm{~Hz}$ hasta $8 \mathrm{kHz}$ del ruido de fondo en el recinto bajo consideración, incluyendo todos los ruidos provenientes del exterior. Se excluyen los ruidos originados por la actividad en el interior del recinto y los ruidos externos atípicos. Posteriormente se comparan los valores del espectro de ruido medido con los valores de los perfiles NC normalizados. Finalmente se le asignará al espectro de ruido, el valor NC del perfil normalizado de mayor valor que es alcanzado por el espectro del ruido en cualquiera de sus bandas.

Por otra parte, los perfiles RC están basados en los valores de nivel de presión sonora originados por sistemas de ventilación y aire acondicionado (calefacción y/o refrigeración), incluyendo ruidos provenientes del exterior obtenidos para las frecuencias centrales de las octavas que van de $16 \mathrm{~Hz}$ a $4 \mathrm{kHz}$ e integradas por dos descriptores:

Primer descriptor. Está determinado por un número que representa el nivel del espectro de interferencia de la palabra (SIL, por su sigla en inglés), obtenido como el promedio aritmético de los niveles de ruido en las octavas de $500 \mathrm{~Hz}, 1 \mathrm{kHz}$ y $2 \mathrm{kHz}$.

Segundo descriptor. Consiste en una letra que indica la calidad del sonido descripta por un observador, calificados como Neutral (N), Ruido de Baja Frecuencia (R), Ruido de Alta Frecuencia (H), Vibraciones Perceptibles, Acústicamente Inducidas (RV). Por razones de espacio, no se describe el procedimiento completo de cálculo y evaluación de los perfiles RC. Por otra parte, cabe mencionar que la norma NBR 10152 incluye en sus recomendaciones el criterio NC.

Para la presente muestra de recintos se realizó la evaluación de los niveles del espectro de ruido de fondo según los criterios de las normas mencionadas anteriormente (Longoni et al, 2014). En la Tabla 3 se presentan los valores de perfil NC y RC recomendados para aulas (mencionadas de manera general en la norma) y en la Tabla 4 los valores asignados a la muestra.

\begin{tabular}{|c|c|c|}
\hline Norma & NC & RC \\
\hline IRAM 4070 & $25-30$ & $25-30$ (N) \\
\hline NBR 10152 & $35-45$ & No especifica \\
\hline
\end{tabular}

Tabla 3 - Valores de perfil NC y RC recomendados.

\begin{tabular}{|c|c|c|c|}
\hline Recinto & NC & \multicolumn{2}{|c|}{ RC } \\
\hline AU11 & 31 & 42 & $\mathrm{H}$ \\
\hline AU154 & 36 & 44 & $\mathrm{~N}$ \\
\hline
\end{tabular}




\begin{tabular}{|c|c|c|c|}
\hline AU225 & 41 & 48 & $\mathrm{H}, \mathrm{RV}$ \\
\hline AU405 & 42 & 51 & $\mathrm{RV}$ \\
\hline AU608 & 30 & 48 & $\mathrm{~N}$ \\
\hline AUD1 & 30 & 46 & $\mathrm{~N}$ \\
\hline AUD2 & 27 & 32 & $\mathrm{~N}$ \\
\hline
\end{tabular}

Tabla 4 - Valores medidos de perfil NC y RC.

Se observa que el 56,2 \% cumple con el perfil NC-30 que establece la norma IRAM 4070. Por otro lado, comparando los valores NC obtenidos contra los criterios de la norma NBR 10152, resulta que el $67,4 \%$ de las aulas cumple con los criterios de confort acústico y el $32,6 \%$ restante presenta valores NC aceptables por ser ésta última menos exigente que la primera.

Según los criterios subjetivos de caracterización del ruido de fondo establecidos en la norma IRAM 4070, para perfiles RC el 61,8 \% presenta un espectro de calidad N mientras que el AU11 de calidad H, el AU405 calidad RV y el AU225 presenta un perfil de calidad H combinado con RV. Cabe destacar que la característica RV observada en el AU405 no se debe al espectro de ruido producido por el SVyAA sino al producido por el tráfico vehicular en su cercanía.

La Fig. 17-a ilustra la distribución de los valores NC y en la Fig. 17-b se presenta la distribución de los valores RC de la muestra.

Se observa que el AUD2 presentó un valor RC 32 y el AU405 RC 51, siendo el más bajo y más elevado de la población respectivamente.

Además, se realizó el análisis de niveles espectrales de ruido de fondo entre las bandas de $125 \mathrm{~Hz}$ y $4 \mathrm{kHz}$. Primero se tomó la diferencia entre el nivel espectral con SVyAA encendido y apagado, para cada banda de octava en el rango de frecuencias mencionado.

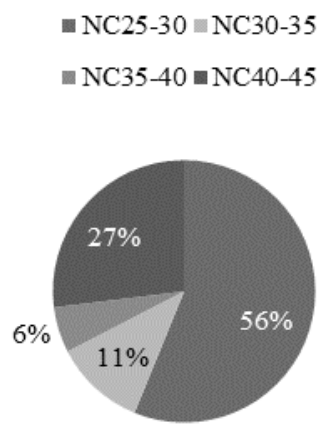

(a)

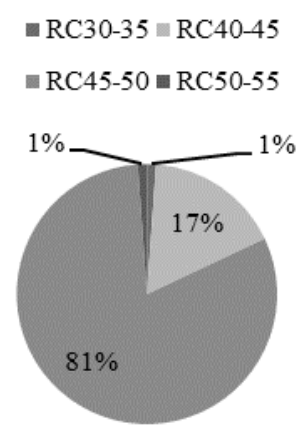

(b)

Fig. 17 - Distribución de los valores NC (a) y RC (b). 
acotado entre las octavas centradas en $125 \mathrm{~Hz}$ y $4 \mathrm{kHz}$.

En la Tabla 5 se presentan los resultados de este análisis. Se observa que para este parámetro el 43,8 \% presenta una diferencia de 15 a $20 \mathrm{~dB}$ y el 55,1 \% de 20 a $25 \mathrm{~dB}$. La menor diferencia encontrada fue de 12,2 dB en el AUD2, el cual está equipado con un sistema de aire acondicionado para conductos.

\begin{tabular}{|c|c|}
\hline Recinto & $\begin{array}{c}\mathrm{L}_{\mathrm{eq}(125 \mathrm{~Hz}-4 \mathrm{kHz})} \\
{[\mathrm{dB}]}\end{array}$ \\
\hline AUD2 & 12,2 \\
\hline AU405 & 16,0 \\
\hline AU225 & 17,3 \\
\hline AU154 & 17,3 \\
\hline AU11 & 18,2 \\
\hline AUD1 & 23,5 \\
\hline AU608 & 23,9 \\
\hline
\end{tabular}

Tabla 5 - Valores del análisis espectral.

\section{ANÁLISIS Y DISCUSIÓN}

\section{Parámetros acústicos derivados de la RIR}

Dentro de la evaluación del tiempo de reverberación se observó que el 98 \% de la población supera el valor recomendado para la frecuencia de $2 \mathrm{kHz}$, siendo esta una de las componentes espectrales más significativas en la inteligibilidad del habla.

Las recomendaciones existentes no hacen referencia al estado de ocupación del aula. Sin embargo a través de mediciones en dos condiciones de ocupación se logró evidenciar que los parámetros acústicos derivados de la RIR se ven afectados significativamente para un factor de ocupación del 60 \% (Cravero et al, 2014). En el caso particular del T30 se obtuvieron disminuciones de un $50 \%$ en $\mathrm{AF}$.

Este último es un aspecto relevante ya que por los resultados obtenidos, es posible afirmar que el campo sonoro varía significativamente en función del grado de ocupación y por lo tanto para obtener un resultado de diseño óptimo, es indispensable considerar el efecto de las personas que ocupan el recinto en su uso habitual además de la condición más desfavorable (ocupación mínima).

\section{Ruido de Fondo}


Como se mencionó anteriormente actualmente nuestro país carece de normativas que establezcan recomendaciones acerca de los niveles globales aceptables en ambientes educacionales específicos y en función de sus volúmenes.

Al comparar los valores obtenidos con los recomendados por la Norma NBR 10152 se observa un bajo cumplimiento de su criterio de confort acústico, el 12,4 \%, mientras que el resto de la población presentó valores aceptables.

Al comparar con criterios más exigentes como los de ANSI S12.60 o BB93 se observa una mala condición en general. El 27,0 \% de la población excede en hasta $15 \mathrm{dBA}$ el límite de ruido de fondo recomendado cuando los SVyAA están apagados. La situación es aún más desfavorable cuando los SVyAA están encendidos, con excesos de hasta $20 \mathrm{dBA}$ en el 32,6\% de la población y de hasta 25 dBA en el 55,1\%.

Comparando los niveles de ruido de fondo con los SVyAA encendidos con la recomendación de la NBR 10152, se observa que el $12 \%$ es considerado aceptable. Mientras que el resto es calificado como no aceptable, con excesos de hasta $5 \mathrm{dBA}(33 \%)$ y de hasta $10 \mathrm{dBA}(55 \%)$.

Por otra parte, ninguno de los recintos estudiados cumple con las recomendaciones de la norma IRAM 4070 según el criterio RC, el cual considera el ruido generado por el SVyAA. El $56,2 \%$ de la población cumple con las recomendaciones de la norma en cuanto a perfiles NC, pero cabe recordar que este criterio solamente evalúa el ruido proveniente del exterior del recinto.

Se puede afirmar que encender el SVyAA en la muestra estudiada es una situación que no pasa desapercibida. Cuando se analizan niveles sonoros globales, el 11,2 \% de la población presenta un aumento de entre 10 y $15 \mathrm{dBA}$ en el ruido de fondo y el 55,1\% entre 15 y $20 \mathrm{dBA}$.

Según el parámetro Leq (125 Hz-4 kHz) obtenido previamente, el $55 \%$ presenta una diferencia de entre 20 y $25 \mathrm{~dB}$ en el rango de frecuencias más importante para la comprensión del habla, lo cual afecta significativamente su inteligibilidad.

\section{Relación Señal Ruido}

Para efectuar un análisis del efecto del nivel de ruido de fondo dentro de un aula, se consideraron como referencia tres niveles de voz: alta (VA), normal (VN) y baja (VB) (Carrión, 2001). Por otra parte, según las recomendaciones de ANSI S12.60, para obtener un grado aceptable de inteligibilidad de un mensaje hablado se requiere que el nivel sonoro del mensaje se encuentre $15 \mathrm{~dB}$ por encima del nivel de ruido de fondo del recinto

La variación de la relación señal-ruido (RSR), medida a $1 \mathrm{~m}$ del orador, para la condición de SVyAA apagado se muestra en la Fig. 18 y en la Fig. 19 para SVyAA encendido, para los tres niveles de voz mencionados.

Por comparación de las Fig. 18 y 19 se observa que en el AUD2 el funcionamiento del SVyAA reduce la RSR en 5 dB. En el AU608 y el AUD1 se observa una reducción de 15 dB, mientras que en el resto de los recintos la reducción de la RSR es de $10 \mathrm{~dB}$.

En el AUD2, la RSR para los 3 niveles de voz se mantiene por encima de $+15 \mathrm{~dB}$ para ambas condiciones del SVyAA. En contraste, en el AU405 las RSR mayor a +15 dB se consigue solamente con VA para la condición de SVyAA apagado.

Para la condición de SVyAA encendido, la RSR en los recintos AU225, AU405 y AU608 (representando el 80,9 \% de la población) queda por debajo de $+15 \mathrm{~dB}$ para los tres niveles de voz considerados. 


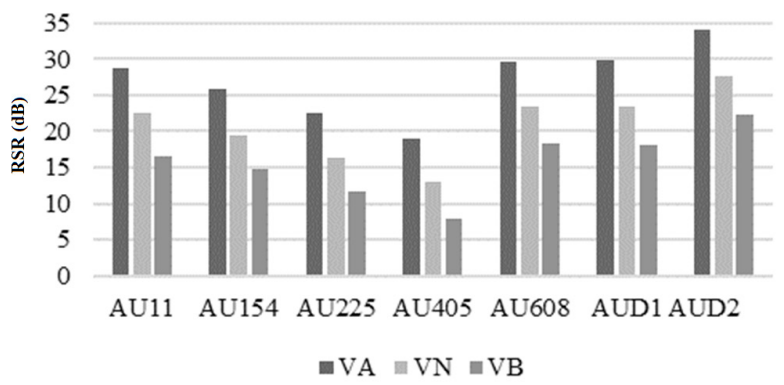

Fig. 18 RSR con SVyAA apagado para cada recinto.

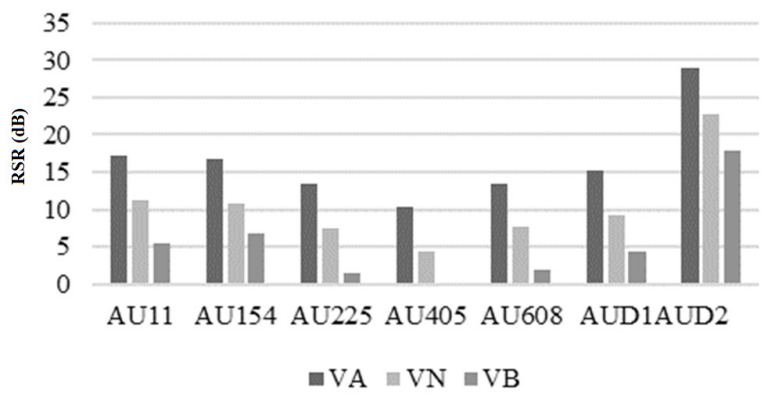

Fig. 19 - RSR con SVyAA encendido para cada recinto.

\section{CONCLUSIONES}

A partir del análisis realizado se demuestra que el 80,9 \% de aulas utilizadas para el dictado de carreras de grado no presentan las condiciones adecuadas para el desarrollo del proceso de enseñanza-aprendizaje.

En general, las aulas durante su diseño carecieron de criterios acústicos. Los materiales constructivos utilizados presentan bajos coeficientes de absorción generando elevados valores de T30. La inadecuada distribución de los modos propios, produce desvíos significativos en los parámetros energéticos derivados de la RIR (C50, D50) en el espectro de BF.

Los SVyAA son instalados sin los cuidados necesarios para que su funcionamiento tenga un efecto despreciable en cuanto al ruido que producen. El estudio realizado evidencia como un sistema SVyAA puede afectar la inteligibilidad del habla reduciendo la RSR significativamente.

Por otra parte los auditorios y aulas, utilizadas en actividades de posgrado y extensión universitaria, presentan mejoras significativas en cuanto a los parámetros derivados de la RIR y ruido de fondo, sin embargo no alcanzan a cumplir con los valores recomendados en diversas normas.

A nivel nacional no existen normas específicas sobre calidad acústica de aulas, exceptuando el caso de IRAM 4070 que, sin ser específica para procesos de enseñanza-aprendizaje, establece valores de referencia para el espectro de ruido de fondo para recintos de 
diversas finalidades entre los cuales se menciona el caso de aulas.

\section{TRABAJO A FUTURO}

Actualmente, se está trabajando en el estudio subjetivo de las características del campo sonoro para la muestra seleccionada mediante la utilización y desarrollo de herramientas de realidad acústica virtual.

Por otro lado, será necesario implementar procedimientos para la medición de la inteligibilidad de la palabra, planteando la metodología adecuada para el análisis de los resultados.

\section{AGRADECIMIENTOS}

A la Secretaria de Ciencia, Tecnología y Posgrado de la UTN. Al Centro de Investigación y Transferencia en Acústica (CINTRA), UA CONICET, de la UTN FRC. 


\section{REFERENCIAS}

Ercoli L., Azzurro A., Méndez A., Stornini A., (2001) "Case study: The acoustical characteristics of typical Argentinean classrooms", Building Acoustics, 8 (4), 301-310, . World Health Organization , (2001) (WHO), Noise in schools, Geneva.

ISO 3382-2:2008, (2008) Acoustics-Measurements of room acoustics parameters - Part 2: Reverberation time in ordinary rooms..

Cravero G. A., Ferreyra P. S., Flores M. D., Budde L., Longoni H. C., Ramos O. A., Tommasini F. C., "Medición y simulación de tiempo de reverberación y otros parámetros acústicos de aulas”, Mecánica Computacional, 32 (34), 2889-2900, (2013).

Cravero G. A., Ferreyra P. S., Longoni H. C., Flores M. D., Ramos O. A., Tommasini F. C., "Análisis espacial de tiempo de reverberación en aulas y auditorios universitarios" Mecánica Computacional, 33 (8), 523-537, (2014).

Longoni H. C., Ferreyra P. S., Cravero G. A., Flores M. D., Ramos O. A., Tommasini F. C., "Evaluación objetiva del espectro del ruido de fondo en aulas universitarias" Mecánica Computacional, 33 (8), 569-583, (2014).

Ferreyra P. S., Esquinas P. B., Orecchia I., Elías M., "Diseño y construcción de una cadena de instrumentación electroacústica para la medición de la respuesta al impulso de recintos según norma ISO 3382”, Mecánica Computacional, 28 (2), 63-72, (2009).

Schroeder M. R., "New method of measuring reverberation time", Journal Acoustical Society of America, 37, 409-412, (1965).

ANSI S12.60, "Acoustical Performance Criteria, Design Requirements, and Guidelines for Schools”, (2002).

Jordan V. L., “A group of objective criteria for concert hall”, Applied Acoustics, 14, 253-266, (1981).

Reichardt, W., Abel Alim, O. \& Schmidt, W., "Definition und Messgrunglage eines objectiven Masses zur Ermittlungder Grenze zwischen brauchbarer und unbrauchbarer Dutrchsichtigkeit bei musikdarbietung”, Acustica, 32 (3), 126-137, (1975).

Thiele R., "Richtungsverteilung und zeitfolge der schallrückwürfe in räumen", Acustica, 3 (2), 291-302, (1953).

Kürer R., "Zur gewinnung von eizahlkriterien bei impulsmessugen in der raumakustik". Acustica, 21, 370-372, (1969).

Ando Y., "Concert Hall Acoustics”, 1st Edition, Springer-Verlag, Germany, 65-68, (1985).

Building Bulletin 93. Acoustic design of schools: A design guide. Stationery Office Books, (2003).

NBR 10152. Niveles de ruido para confort acústico. (1987).

IRAM 4070. Ruidos. Procedimiento para su evaluación utilizando los perfiles NC y RC. (2008). 
Cravero G. A., Ferreyra P. S., Longoni H.C., Flores M. D., Ramos O.A., y Tommasini F. C., "Analisis de parámetros acústicos de recintos para dos condiciones de ocupación: estudio de caso de un aula universitaria", Actas del IX Congreso Iberoamericano de acústica - FIA 2014, Valdivia - Chile, 1 al 3 de diciembre de 2014, 223-231, (2014).

Carrión Isbert, A. “Diseño acústico de espacios acústicos arquitectónicos”, Primera edición, España, pág. 46, (1998). 